\title{
Cervical Lesion: Types and Etiology
}

\section{FASOGBON Samuel A1*, ADEBAYO Ahmed 0², AJILEYE Ayodeji B³,} OKORIE Nnaemeka ${ }^{4}$ and ADELUWOYE Adekunle $0^{5}$

${ }^{1}$ Public Health In-vitro Diagnostic Control Laboratory, Medical Laboratory Science Council of Nigeria, Yaba-Lagos, Nigeria

${ }^{2}$ Department of Medical Laboratory Science, Lagos State College of Health Technology,

Yaba-Lagos, Nigeria

${ }^{3}$ Department of Medical Laboratory Science, Achievers University Owo, Ondo State, Nigeria

${ }^{4}$ Department of Medical Laboratory Science, Faculty of Health Sciences and Technology, Taraba State University, Jalingo, Nigeria

${ }^{5}$ Department of Medical Laboratory Science, Lead City University, Ibadan, Oyo State, Nigeria

*Corresponding author: Fasogbon, Samuel Ayobami, Public Health In-vitro Diagnostic Control Laboratory, Medical Laboratory Science Council of Nigeria, 8 Harvey Road, Yaba, Lagos State, Nigeria, Tel: +2348032229904; Email: samfash4best@gmail.com

\section{Abstract}

A cervical lesion is an area of abnormal tissue found on the cervix, which is the lower end of a woman's uterus. The type of the lesion can be atypical squamous cells, squamous intraepithelianeoplasia, and atypical glandular cells. The early stages of cervical cancer may be asymptomatic. In advanced disease, there may be metastases to the abdomen, lungs or any other organ. Infection with some types of human papilloma virus (HPV) is the highest risk factor for cervical cancer which can be transmitted due to multiple sex partners, followed by smoking. Other risk factors include human immunodeficiency virus (HIV), early age at first sexual intercourse, early age at first pregnancy, tobacco use, exposure to passive smoke and other related factors. It is therefore recommended that all the risk factors should be avoided to reduce cervical cancer in the society and women should go for regular cervical screening as early detection can easily be treated.

Keywords: Cervical Lesion, Etiology, HPV, Smoking

Abbreviations: HIV: Human Immunodeficiency Virus; HPV: Human Papillomavirus; AGC: 5.3. Atypical Glandular Cells; SIL: Squamous Intraepithelial Lesion; STD: Sexually Transmitted Disease; DES: Drug Diethylstilbestrol.

\section{Introduction}

A cervical lesion is an area of abnormal tissue found on the cervix, which is the lower end of a woman's uterus. Such lesions may be found during routine Pap smears and can point the presence of infection with human 
papillomavirus (HPV) and/or precancerous changes in the cervix cells [1-3].

A precancerous cervical lesion, which can otherwise called an intraepithelial lesion, is an abnormality in the cervical cells that could later develop into cervical malignancy. This abnormality found in cervical cells may develop into cervical cancer if left untreated $[1,2]$.

\section{Types of Cervical Lesions}

There are two main types of cervical cells, squamous and glandular, and abnormalities can occur in either type [1]. The most common types of precancerous cervical lesions include:

\section{Atypical Squamous Cells}

Atypical squamous cells mean that abnormalities have been detected in the squamous cells of the cervix. This can indicate human papillomavirus (HPV) infection, other infections, or possibly precancerous cells of another cause. Further diagnosis may be required to determine what the specific abnormalities. In some cases, this may mean a repeat Pap test in a few months (maybe after 12months). A Pap test with this kind of finding may be reported as "atypical squamous cells of uncertain significance," abbreviated as ASCUS [1]. But it's not clear if the changes are caused by HPV infection. Other things can cause cells to look abnormal, such as irritation, some infections, such as a yeast infection, growths, such as polyps or cysts that are benign (not cancer), and changes in hormones that occur during pregnancy or menopause [2].

\section{Squamous Intraepithelial Lesion (SIL)}

This lesion means that there are changes on the cervix that may be precancerous. SIL lesions are classified as either low-grade (LSIL), LSIL is sometimes called mild dysplasia. It may also be called CIN 1 or high-grade (HSIL), high-grade lesions are more likely to progress to malignancy of the cervix, and HSIL is sometimes called moderate or severe dysplasia. It may also be called CIN 2 , CIN $2 / 3$, or CIN 3. HSIL means that there are more serious changes than LSIL, in cervical cells [1-2].

\section{Atypical Glandular Cells (AGC)}

These may indicate a possible precancerous lesion in the upper area of the cervix or inside the uterus [1]. AGC means that some glandular cells were found that do not look normal. More testing is usually recommended. Colposcopy and biopsy as described in the follow-up testing section [2].

\section{Signs and Symptoms}

The early stages of cervical cancer may be asymptomatic $[4,5]$. Vaginal bleeding, contact bleeding, or (rarely) a vaginal mass may indicate the presence of malignancy. Moderate pain during sexual intercourse and vaginal discharge are symptoms of cervical cancer. In advanced disease, there may be metastases to the abdomen, lungs or any other organ. Advanced cervical cancer may include: loss of appetite, weight loss, fatigue, pelvic pain, back pain, leg pain and swollen legs, heavy bleeding from the vagina, bone fractures, and/or (rarely) leakage of urine or faces from the vagina [6].

\section{Etiology}

Cells infected with the HPV virus heal on their own in most cases. In some cases, however, the virus continues to spread and becomes an invasive cancer. Cervix in relation to upper part of vagina and posterior portion of uterus., showing difference in covering epithelium of inner structures. The causes of cervical malignancy include:

Viral Infection (Commonly Human Papilloma Virus): Infection with some types of human papilloma virus (HPV) is the highest risk factor for cervical cancer, followed by smoking [7]. Other risk factors include human immunodeficiency virus (HIV). Not all of the causes of cervical cancer are known, however, and several other contributing factors have been implicated [8].

Human papillomavirus type 16 and 18 are the cause of $75 \%$ of cervical cancer globally while 31 and 45 are the cause of another $10 \%$ [9].

Women who engage in many sexual partners (or who have sex with men who have had several other partners) have a greater risk [10,11]. Of the 150-200 types of HPV known [12], 15 are categorized as high-risk types $(16,18$, $31,33,35,39,45,51,52,56,58,59,68,73$, and 82$), 3$ as probable high-risk $(26,53$, and 66$)$, and 12 as low-risk (6, $11,40,42,43,44,54,61,70,72,81$, and CP6108) [13].

\section{Smoking}

Also linked to the development of cervical cancer is smoking $[14,15,7]$. There are a few separate ways that smoking can increase the risk of cervical cancer in women which can be by direct and indirect methods of inducing cervical cancer $[14,16]$. A direct way of contracting this cancer is that a female smoker has a higher chance of developing cervical intraepithelial neoplastic 3 (CIN3) which has the high potential of becoming cervical cancer [14]. When CIN3 lesions lead to cancer, most of them have the help of the HPV virus, but that is not always the case 
which is why it can be considered a direct link to cervical cancer. Heavy smokers and long term smokers seem to have high risk of getting the CIN3 lesions than lighter smokers or non-smokers [7]. Although smoking has been linked to cervical cancer, it aids in the development of HPV which is the leading cause cervical cancer. Also, not only does it aid in the development of HPV, but if the woman is already HPV-positive she is at an even greater likelihood of contracting cervical cancer $[7,17]$.

$>\quad$ Other Risk Factors Includes [18]:

- Early age at first sexual intercourse (17 years or younger)

- Early age at first pregnancy

- Tobacco use, including exposure to passive smoke

- Low socioeconomic status (lack of screening)

- History of any sexually transmitted disease (STD), especially HPV and human immunodeficiency virus (HIV)

- History of multiple sex partners (five or more)

- History of childhood sexual abuse

- Intimate partner abuse

- Women whose mothers used the drug diethylstilbestrol (DES) during pregnancy

- Hormonal contraceptive use

- Ethnic background- African-American women experience a $72 \%$ higher incidence compared with Caucasian women

- High parity (number of births)

- Alcohol and drug use (impaired decision making) [18]

\section{Conclusion}

A cervical lesion is the abnormal tissue that is found on any area of the cervix. The type of the lesion can be atypical squamous cells, squamous intraepithelianeoplasia, and atypical glandular cells. Such lesions may be found during routine Pap smear screening and can indicate the presence of human papillomavirus (HPV) infection and/or precancerous changes in the cells of the cervix. The causes of the cervical lesion can be viral (Most likely HPV), smoking, and other related risk factors.

\section{Recommendations}

The following are therefore recommended to reduce cervical cancer in the society.

- that women should go for cervical screening every 6 months at least as early detection of cervical abnormalities can quickly be treated

- avoid multiple sex partners which are the lead risk of getting infected with HPV
- Early age sexual intercourse (17 years or younger) should be avoided and prevented

- Smoking, Tobacco use, including exposure to passive smoke should be avoided

- avoid the use of Hormonal contraceptive

- Moderate Alcohol intake

\section{References}

1. MediResource (2014) cervical cancer diagnosis. MediResource Inc. MediResource reaches millions of Canadians each year.

2. NCI (2018) Understanding cervical changes: Next Steps After an Abnormal Screening Test. National Cancer Institute.

3. NCI (2014) Cervical Cancer Prevention (PDQ®). National Cancer Institute.

4. Kumar V, Abbas AK, Fausto N, Mitchell RN (2007) Robbins Basic Pathology. 8th (Edn.), Saunders Elsevier, pp: 718-721.

5. Holland-Frei cancer medicine (2009) Cerval lesions $8^{\text {th }}$ (Edn.), McGraw-Hill Medical, New York, pp: 1299.

6. Nanda R (2006) Cervical cancer: MedlinePlus Medical Encyclopedia. National Institutes of Health.

7. Gadducci A, Barsotti C, Cosio S, Domenici L, Riccardo GA (2011) Smoking habit, immune suppression, oral contraceptive use, and hormone replacement therapy use and cervical carcinogenesis: A review of the literature. Gynecological Endocrinology 27(8): 597604.

8. Stuart C, Ash M (2006) Gynaecology by Ten Teachers. 18 (Edn.), Hodder Education.

9. Dillman (2009) Principles of cancer biotherapy. In: Robert K, Oldham, Robert O, (Eds.), 5th (Edn.), Springer. Dordrecht, pp: 149.

10. American Cancer Society (2010) Detailed Guide: Cervical Cancer. Can cervical cancer be prevented?

11. Marrazzo JM, Koutsky LA, Kiviat NB, Kuypers JM, Stine K (2001) Papanicolaou test screening and prevalence of genital human papillomavirus among women who have sex with women.Am J Public Health 91(6): 947-952.

12. Gottlieb N (2002) A Primer on HPV. Benchmarks. National Cancer Institute. 
13. Muñoz N, Bosch FX, de Sanjosé S, Herrero R, Castellsagué $X$, et al. (2003) Epidemiologic classification of human papillomavirus types associated with cervical cancer. N EnglJ Med 348(6): 518-527.

14. Luhn P, Walker J, Schiffman M, Zuna RE, Dunn ST, et al. (2013) The role of co-factors in the progression from human papillomavirus infection to cervical cancer. Gynecologic Oncology 128(2): 265-270.

15. Remschmidt C, Kaufmann AM, Hagemann I, Vartazarova E, Wichmann O, et al. (2013) Risk Factors for Cervical Human Papillomavirus Infection and High-Grade Intraepithelial Lesion in Women Aged 20 to 31 Years in Germany. International Journal of Gynecological Cancer 23(3): 519-526.

16. Agorastos T, Miliaras D, Lambropoulos AF, Chrisafi S, Kotsis A, et al. (2005) Detection and typing of human papillomavirus DNA in uterine cervices with coexistent grade I and grade III intraepithelial neoplasia: biologic progression or independent lesions?". European Journal of Obstetrics \& Gynecology and Reproductive Biology 121(1): 99103.

17. Jensen KE, Schmiedel S, Frederiksen K, Norrild B, Iftner T, et al. (2012) Risk for cervical intraepithelial neoplasia grade 3 or worse in relation to smoking among women with persistent human papillomavirus infection. Cancer Epidemiology, Biomarkers \& Prevention: a Publication of the American Association for Cancer Research, Cosponsored by the American Society of Preventive Oncology 21(11): 1949-1955.

18. Goodman CC, Snyder TE (2012) Differential Diagnosis for Physical Therapists, Screening for Referral. W B Saunders Company. 